\title{
Substantial caries-inhibiting effect of fluoride varnish suggested
}

\author{
How effective are school-based fluoride varnish programmes at preventing \\ approximal caries?
}

\begin{abstract}
Sköld UM, Petersson LG, Lith A, Birkhed D. Effect of schoolbased fluoride varnish programmes on approximal caries in adolescents from different caries risk areas. Caries Res 2005; 39:273-279
\end{abstract}

Design A randomised controlled trial (RCT) was carried out over a period of 3 years. In total, 758 adolescents (age 13 years at baseline) from three areas in Sweden, from low, medium and high caries-risk areas, were recruited into the study. Bitewing radiographs were taken at baseline and at the end of the trial period by the participants' own dentists. Radiographic caries recording was performed, blinded, by one of the authors.

Intervention Three fluoride varnish regimes were employed over a 3-year period, applied as follows: twice a year at 6-month intervals (Group 1, six applications in total); three times a year all within 1 week (Group 2, nine applications in total); once a month during school terms (Group 3, 24 applications in total). All approximal surfaces were treated with a total of $0.3 \mathrm{ml}$ fluoride varnish. Participants were advised to refrain from eating or toothbrushing on the day. A control group received no fluoride varnish (Group 4).

Outcome measure Approximal caries prevalence after 3 years, incidence, progression and prevented fraction (PF) were measured.

Results Intra-examiner kappa was 0.90 for all scores and 0.82 for carious surfaces. There were no significant differences in caries prevalence after 3 years between groups for dentin lesions and filled surfaces, and for enamel lesions or filled surfaces. For all areas together and in the high-risk area, however, there were significantly more dentin lesions in the control group than in fluoride varnish groups. After 3 years, statistically significant differences $(P<0.001)$ for total caries incidence and new enamel lesions were found between test and control groups in all areas together, in high-risk areas and in medium-risk areas. The largest differences were found in the high-risk area: the control group developed $3.05 \pm 3.37$ (mean \pm SD) lesions compared with $0.54 \pm 1.26$ in Group 3, $0.95 \pm 1.67$ in Group 1 and $1.4 \pm 1.89$ in Group 2. Incidence was also significantly less in Group 3 than in Group 2. For all areas together, fluoride varnish treatment once a month (Group 3) had the best preventive effect with a prevented fraction of $76 \%$ compared to $57 \%$ (Group 1) and $47 \%$ (Group 2). In the mediumand high-risk areas, treatment twice a year (Group 1) resulted in a PF of 66 and $69 \%$ and was the most cost-effective option (results published elsewhere).

Conclusions Monthly fluoride varnish application was the most effective preventive regime in this school-based programme. Sixmonthly fluoride varnish application was the most cost-effective method, however, for high- and medium-risk groups over the 3-year period.

Address for correspondence: Ulla Moberg Sköld, Department of Preventive Dental Care, FTV Södra Bohuslän, Regionens Hus, SE-405 44 Göteborg, Sweden. E-mail: ulla.mobergskold@vgregion.se

\section{Commentary}

This was an interesting Swedish study that tested whether fluoride varnish preventive programmes at school, using Duraphat ${ }^{\mathbb{R}}$ (Colgate, Piscataway, NJ, USA) varnish in various frequencies of application, involving children from different caries risk areas, can be effective in preventing approximal caries in the permanent dentition.

The three geographical sites in the trial were assessed as low, medium and high caries-risk based on differing income, values for decayed/filled teeth in 12-year olds, and natural fluoride concentration in water (optimal levels in the low-risk area only). In general, large effects (in caries incidence) are described for the various frequencies of varnish application in comparison with the control group, especially for the group having the most frequent fluoride-application regimen in the medium and high caries-risk areas.

The authors seem to have made provision for reporting of adverse effects, although no adverse effects were found.

This recent study is in line with the objectives of the Cochrane review on the effectiveness of fluoride varnishes (covering all available experimental evidence on the topic up to 2002, and in the process of being updated), ${ }^{1}$ which examines safety of the intervention and whether the caries preventive effect of varnishes is influenced by initial caries levels and background exposure to fluorides.

The reporting of a new RCT on fluoride varnishes and dental caries is sound - vast numbers of studies were excluded from the relevant Cochrane review mainly on the basis of inadequate methodologies, and those relatively few studies that were included varied in quality.

In this study, in each area and within each school class, 13-yearold children were randomised into three treatment groups and a control group. Although the method of randomisation is not described, it seems to have provided groups with similar caries prevalence in the medium- and high-risk areas; there were significant differences in dentin caries prevalence in the low-risk area which may be considered clinically unimportant.

Distribution of other background or prognostic factors at baseline is not reported by group, and although the overall dropout rate, of $11 \%$, from 854 participants at start, is relatively low after 3 years it is not clear whether differential losses have occurred among groups because numbers at baseline are not reported. Data from each group by area, relate to only those who remained in the study and attended all treatment sessions, and this has the potential to influence findings positively. Outcome assessment was blind, ensuring control of detection bias, and reliability of examination was very good, but participants and dental nurses (applying the intervention) could not have been blinded in a trial with a notreatment control. Although dentists treating the children in the yearly check-ups were unaware of group assignment, co-intervention is likely in the study, as $95 \%$ of all children were treated with one application of fluoride varnish. Although co-intervention can 
bias results in either direction, the possibility of overestimation of a treatment effect in no-treatment control trials has been suggested in relevant Cochrane reviews of topical fluorides, and cannot be overlooked.

When the results of this new study are considered in the light of evidence summarised in the Cochrane review, both suggest a substantial caries-inhibiting effect of fluoride varnish. It should be noted, however, that whereas the new study's PF relate to incidence of new approximal enamel lesions, the Cochrane review combined results from various frequencies of fluoride varnish application, tooth surface types (including approximal surfaces), and considered caries increment at the dentin level. Confidence intervals are not provided in this report and it is not possible to assess how precise the estimates of effects were, but additional information such as this might be available in a another publication on this study, reporting on cost-effectiveness. From that, the authors anticipate that fluoride-varnish treatment at 6-month intervals is the most cost-effective strategy.

\section{Practice point}

- When considered in conjunction with the Cochrane review, results here suggest a substantial caries-inhibiting effect of fluoride varnish.

Valeria Marinho

Centre for Adult Oral Health, Institute of Dentistry, Queen Mary's School of Medicine and Dentistry Turner Street, Whitechapel,

London, UK

1. Marinho VCC, Higgins JPT, Logan S, Sheiham A. Fluoride varnishes for preventing dental caries in children and adolescents. The Cochrane Database of Systematic Reviews 2002.

Evidence-Based Dentistry (2006) 7, 9-10

doi:10.1038/sj.ebd.6400374 